\title{
Analysis on detection probability of satellite-based AIS affected by parameter estimation
}

\author{
Xiaofeng Zhu' ${ }^{1,}$, Wei Wang ${ }^{1}$ \\ ${ }^{1}$ China Academy of Space Technology (Xi'an), Xi'an 710100, China; \\ axfatxd2007@163.com
}

Keywords: satellite-based AIS, detection probability, frequency offset estimation, delay estimation.

\begin{abstract}
In order to analysis marine detection performance of the satellite-based AIS receiving system and provide a reference for related algorithms, the relationship between signal detection bit error rate(BER) and detection probability is analyzed and simulated, besides, the detection BER is simulated at different parameter estimation accuracy. Simulation results show that the energy ratio of any two signals of the mixed signals must be greater than $6 \mathrm{~dB}$ for the receiving system, while the error of the amplitude estimation, frequency offset estimation and delay estimation must not be higher than a value. The simulation model of detection probability introduced can be referenced in the design of the satellite-based receiving system.
\end{abstract}

\section{Introduction}

AIS is abbreviated form of marine automatic identification system, the system combined a global positioning system allows the ship to obtain the dynamic information of other vessels nearby waters so as to improve the safety of navigation, control of maritime information purposes. AIS system uses SOTDMA technology, in a self-organizing region, information collision does not occur, sight communication range of AIS system on ship or land is small, information collision does not occur between adjacent cells. When the AIS system is transferred to the satellite, because the satellite coverage area is relatively large, satellite-based AIS receiving system can simultaneously cover a plurality of self-organizing cells, leading to a collision signal transmitted by different cells occur on the satellite, collision signals overlap at the time domain and frequency domain, thus the detection performance of the conventional receiver greatly reduced.

In order to improve the performance of satellite-based AIS receiving system maximally, Researches in recent day is mainly divided into two directions, one is to improve the satellite reception antenna with the purpose of reducing collisions numbers of the received signals, the other one is to improve detection algorithms of the signal receiver, which try to reduce the signal detection bit error rate(BER) in the condition of signal collision so as to enhance the detection probability of the marine. Several methods on how to improve the antenna was described in [1,2]. An efficient satellite-based AIS signal receiving system was described in [3,4], satellite-based AIS mixed-signals are demodulated one by one by the way of feedback and cancellation in this system, which can sharply enhance the marine detection probability of receiving system.

Based on the system in [3], a modified algorithm is proposed in this paper. Firstly, An analysis model on the relationship between the detection BER and the marine detection probability has been established, Secondly, the impact of the parameter estimation on the detection BER after reconstruction and cancellation was simulated and analyzed, and then we can obtain the requirement of the system designing.

\section{Model of receiving system}

In the recent research, satellite-based AIS receiving systems generally used the method described in $[3,4]$, the receiving system block diagram is shown below 


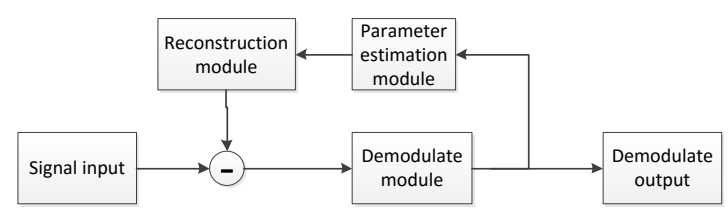

Figrue1 block diagram of the receiving system of satellite-based AIS

In figure 1, the input signal is GMSK baseband mixed-signal, demodulate module demodulates the largest energy GMSK baseband signal, reconstruction module and parameter estimation module completes the reconstruction of the demodulated signal. The main process of this receiving system is, firstly demodulated the biggest energy signal in mixed signals and reconstructed it, then canceled it by subtracting it from mixed signal, and then demodulated the remaining signals in the same manner. Obviously, signal reconstruction performance greatly affects the demodulation performance of mixed signals, the better the signal reconstruction performance is, the better the cancellation performance is, demodulated signal has less effect on the demodulation performance of remaining signals. Thus, the receiving system need to estimate the amplitude, delay, frequency offset and other relevant parameters of the demodulated signal to maximize the performance of signal cancellation .

An analysis on the performance of signal reconstruction for the case of two signals mixed is preferred to be made in this paper, Since the case of much more signals mixed is similar to it, a general expression of the received signal for satellite-based AIS system can be expressed as:

$$
x(t)=A_{1} s_{1}(t) e^{j\left(\tau_{1}+2 \pi f_{d} t\right)}+A_{2} s_{2}(t) e^{j\left(\tau_{1}+2 \pi f_{d} t\right)}+w(t)
$$

In formula (1), $s_{i}(t)$ represents the GMSK baseband modulated signal, $A_{i}$ is the amplitude of signal after the propagation attenuation, $\tau_{i}$ represents the AIS signal propagation delay, and $f_{d}$ is the frequency shift of signal. The frequency shift of signal in the satellite-based AIS system is the Doppler frequency shift caused by the relative motion of the satellite and the marine, since the speed of ship is negligible compared with the speed of the satellite, so the Doppler frequency offset of different AIS signals are approximately equal for the satellite receiver. As it can be seen From (1), in order to cancel one of the mixed signal, it is needed to estimate the amplitude, propagation delay and Doppler frequency shift of the signal. Obviously, the estimation accuracy of the above parameters directly affects the performance of remaining demodulated signal.

\section{Marine detection probability}

Generally, the detection probability of marine refers to the probability of correctly detect of the AIS information transmitted by a ship within the satellite observation time. Marine detection probability was analyzed in [5,6,7]with the restrain of that the receiving system can only detect non-collision AIS signal. These analyses did not consider the detection capability of the reception system to detect collision signal, and not analyze the relationship of the performance of the detection error with marine detection probability.

For most ships at sea, the time of satellite observation is short. During this time, the change of ship protocol information is negligible, so the frame message sent by ships may be considered the same. Therefore, ships are considered to be detected as long as one of all frames message sent by ship is correctly detected within satellite observation period. According to the protocol of AIS system, one frame message includes 16-bit CRC checksum. Thus, whether the frame message is right or not is often determined by the CRC checksum. Based on this principle, the relationship between reception system BER and marine detection probability is simulated in this section. In order to simplify the simulation model, we only consider the case of a single satellite. Some parameters in satellite-based AIS receiving system are simplified as shown in the following table [8-9].

Table 1 some parameters in satellite-based AIS receiving system

\begin{tabular}{cc}
\hline Parameter & Value \\
\hline Satellite altitude $(\mathrm{km})$ & 950 \\
Shipping reporting interval (s) & 6 \\
Observe time of single satellite (s) & 818 \\
\hline
\end{tabular}


The observe time of single satellite will varies with the latitude of shipping, but in the case of a single pass of a single satellite, satellite observation time is almost unchanged.

The number of information in one frame sent by a ship during satellite observation time can be calculated by formula (2).

$$
N_{F}=\frac{T_{s}}{\Delta T}
$$

In formula (2), $N_{F}$ is frame number, $T_{s}$ is satellite observation time, $\Delta T$ is interval of frame transmitted. Suppose that the BER of the receiving system is $P_{e}$, and then frame error rate(FER) $P_{e, F}$ can be obtained by using Monte Carlo method. With above assumptions, the detection probability of a ship can be obtained as following equation.

$$
P_{d}=1-\left(P_{e, F}\right)^{N_{F}}
$$

Figure 2 refers to relationship between receiving system detection BER and marine detection probability. For a certain BER, we obtain the FER through statistic on the result of 10,000 times repeated test, and then use formula (3) to calculate marine detection probability.

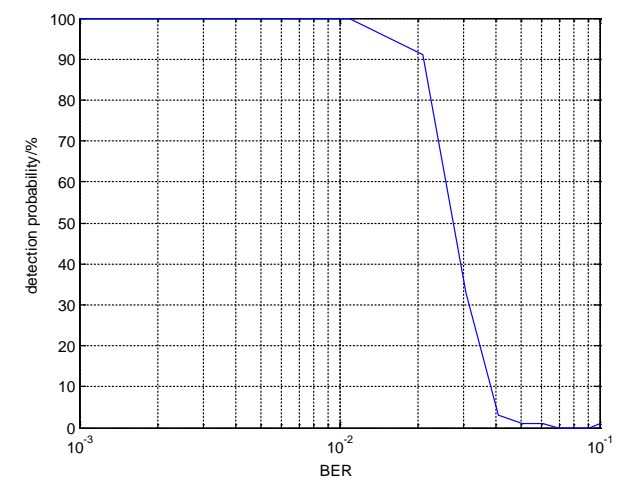

Figure 2 the relationship between receiving system BER and marine detection probability

As is known from figure 2, marine detection probability is up to $100 \%$ when the BER is less than $10-2$. If the BER is over 10-2, the marine detection probability is sharply decreased. When the BER is over $3 * 10-2$, the marine detection probability is low to $30 \%$. Therefore, in order to guarantee the performance of satellite-based AIS receiving system, the BER should be not higher than 10-2.

\section{Parameter estimation}

Know by section 2 analyses, for two mixed signals, the performance of cancelling bigger energy signal will affect demodulation BER of smaller energy signal, and it has a direct relationship with parameter estimation accuracy. This section simulates and analyses the impact on demodulation BER of smaller energy signal by amplitude estimation accuracy, frequency offset estimation accuracy and delay estimation accuracy.

Under conditions of two mixed signals, a non-coherent sequence detection based on Viterbi algorithm is used to detect GMSK signal in [10,11,12], for different ratio of signal energy, the detection BER curves is shown below.

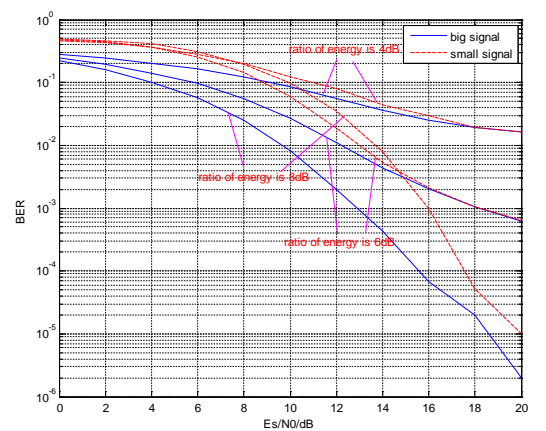

Figure 3 demodulation BER curves of different ratio of signal energy 
Signal to noise ratio referred in figure 3 is the ratio of the energy of bigger signal and the power spectral density of gauss white noise, as can be seen from figure 3:

(1) The demodulation performance of small signal is not as good as that of big signal, the best case is that it just approach that of big signal, thus when the number of mixed signals is much more greater, the signal demodulation performance will gradually decline as the signal energy decline, and due to an increase in number of mixed signals, demodulation performance of the biggest signal is poorer than that in the case of the two mixed signals.

(2) When the ratio of two signal energy is smaller than $6 \mathrm{~dB}$, the detection BER of both signals is higher than $10^{-2}$, analysis of previous section shows that the detection probability of the ship will decline severely under this condition, and then the receiving system will not detect the ship information. Therefore, according to the figure 3, the ratio of any two signals of mixed signals must be bigger than $6 \mathrm{~dB}$ for the receiving system referred in this paper.

In the figure 3, it is assumed big signal is completely canceled without considering the impact of parameter estimation accuracy. The following figures show the BER curves of small signal at different amplitude, frequency, and delay estimation accuracy.
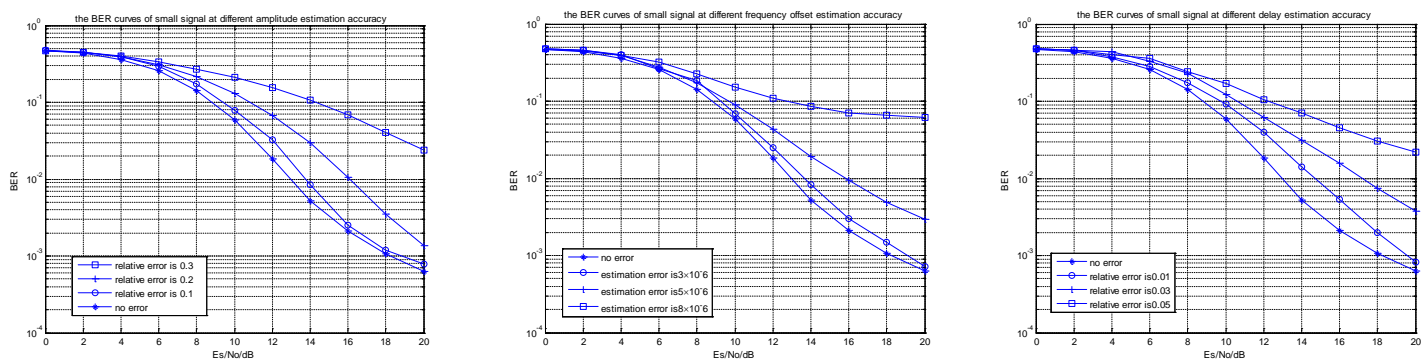

Figure 4 the BER curves of small signal at different parameter estimation accuracy

In figure 4 , relative error of amplitude estimation is defined as $\Delta A=\frac{|A-\bar{A}|}{A}$, and $A$ is the real amplitude of the signal, $\bar{A}$ is the estimated value. Error of frequency offset estimation is defined as $\Delta f_{d}=\frac{\left|f_{d}-\bar{f}_{d}\right|}{T_{s}}$, and $f_{d}$ is the real frequency offset of the signal, $\bar{f}_{d}$ is the estimated value. Relative error of delay estimation is defined as $\Delta \tau=\frac{|\tau-\bar{\tau}|}{2 \pi}$, and $\tau$ is the real delay of the signal, $\bar{\tau}$ is the estimated value. As can be seen in the figure 4, the demodulation BER of small signal is significantly affected by the parameter estimation accuracy, in order to obtain better performance of demodulation, the relative error of amplitude estimation must be less than 0.2 , the error of frequency offset estimation must be less than $5 \times 10^{-6}$, the relative error of delay estimation must be less than 0.03 . The above simulation takes into account only a single parameter estimation, and the parameter estimation accuracy should be further improved in the actual situation.

\section{Summary}

Based on some parameters of the actual satellite-based AIS system, this paper establishes a relationship between detection probability and detection BER of the receiver. Besides, this paper also simulates and analysis the performance of demodulation at different parameter estimation accuracy after that the demodulated signal is canceled. Simulation results show that AIS information transmitted by the ship cannot be detected correctly when the demodulation BER is higher than $10^{-2}$, thus the energy ratio of any two signals of the mixed signals must be greater than $6 \mathrm{~dB}$ for the receiving system, and simulation results also can be used as a reference for parameter estimation accuracy before the receiving system being designed. Thus, this paper is useful and instructive for designing of the satellite-based AIS receiving system. 


\section{References}

[1]Ole Fredrik Haakonsen Dahl. Space-Based AIS receiver for maritime traffic monitoring using interference cancellation[D]. Norwegian University of science and technology department of electronic and telecommunications, June 2006.

[2]Miguel A.Cervera, Alberto Ginesi. On the Performance Analysis of a Satellite-based AIS System. IEEE 10th International Workshop on Signal Processing for Space Communication,2008,pp:1-8.

[3]Paolo Burzigotti, Alberto Ginesi, Giulio Colavolpe. Advanced Receiver Design for Satellite-Based AIS Siganal Detection. IEEE 5th Advanced Satellite Multimedia Systems Conference and the 11th Signal Processing for Space Communications Workshop,2010,pp:1-8.

[4]Giulio Colavolpe, Tommaso Foggi, Alessandro Ugolini. A highly efficient receiver for satellite-based automatic identification system signal detection. 2014 7thASMS/13thSPSC,2014.

[5]CHENG Yun, CHEN Lihu, Chen Xiaoqian. Modeling and simulation analysis of detection probability for space-based AIS. Journal of national university of defense technology,2014,36(3).

[6] ZHONG Jie, WANG Huai-sheng, ZHENG Li. Analysis and simulation of ship detection probability of space-based AIS. Telecommunication Engineering,2010,50(10).

[7]WANG Hai-yan, XI Zia-jie, YANG Wen-jing. Calculation model and algorithm analysis of detection probability of space-based AIS. Telecommunication Engineering,2011,51(11).

[8]ITU-R M.2084 report,2006

[9] XU Bin, RUI Guosheng, ZHANG song. Development and perspective of space-based AIS.

[10]Giulio Colavolpe, Riccardo Raheli. Noncoherent Sequence Detection. IEEE Trans. On Communication.1999,9,pp:1376-1385

[11] Giulio Colavolpe, Riccardo Raheli. Non-coherent Sequence Detection of M-ary PSK. IEEE International Conference on Communications.1997,pp:21-25

[12] J.P.Fonseka. Noncoherent detection with Viterbi decoding for GMSK signals. IEE ProceedingsCommunication.2002,pp:373-379 